\title{
Philosophiques
}

\section{Lecture d'Althusser}

\section{Daniel Goldstick}

Volume 12, numéro 2, automne 1985

URI : https://id.erudit.org/iderudit/203291ar

DOI : https://doi.org/10.7202/203291ar

Aller au sommaire du numéro

Éditeur(s)

Société de philosophie du Québec

ISSN

0316-2923 (imprimé)

1492-1391 (numérique)

Découvrir la revue

\section{Citer cet article}

Goldstick, D. (1985). Lecture d'Althusser. Philosophiques, 12(2), 363-391.

https://doi.org/10.7202/203291ar

\section{Résumé de l'article}

La cohérence du marxisme de Louis Althusser est ici assaillie à partir de la théorie de la connaissance engelsienne. On prétend dévoiler certains " concepts »-clés de l'althusserisme comme étant des métaphores non susceptibles de toute interprétation qui se tienne. Son adhésion foudroyante à l'objectivisme réaliste se manifeste comme fausse (quand même sincère). Cinq problématiques althusseriennes sont examinées et critiquées successivement. Il s'agit de sa désapprobation du concept engelsien de vérité relative et objective, sa distinction entre l'objet de connaissance et l'objet réel, sa conception de «l'idéologie », sa conception de « l'empirisme », et sa conception de la philosophie.
Ce document est protégé par la loi sur le droit d'auteur. L'utilisation des services d'Érudit (y compris la reproduction) est assujettie à sa politique d'utilisation que vous pouvez consulter en ligne.

https://apropos.erudit.org/fr/usagers/politique-dutilisation/ 
PHILOSOPHIQUes, Vol. XII, Numéro 2, Automne 1985

\title{
LECTURE D'ALTHUSSER*
}

\author{
par Daniel Goldstick
}

\begin{abstract}
RÉSUMÉ. La cohérence du marxisme de Louis Althusser est ici assaillie à partir de la théorie de la connaissance engelsienne. On prétend dévoiler certains « concepts »-clés de l'althusserisme comme étant des métaphores non susceptibles de toute interprétation qui se tienne. Son adhésion foudroyante à l'objectivisme réaliste se manifeste comme fausse (quand même sincère). Cinq problématiques althusseriennes sont examinées et critiquées successivement. Il s'agit de sa désapprobation du concept engelsien de vérité relative et objective, sa distinction entre l'objet de connaissance et l'objet réel, sa conception de "l'idéologie ", sa conception de "l'empirisme », et sa conception de la philosophie.
\end{abstract}

ABSTRACT. The coherence of Louis Althusser's "Marxism" is here assailed from the standpoint of Engels' theory of knowledge. Key Althusserian "concepts" turn out to be mere metaphors insusceptible of any consistent construal, it is claimed, while his blustering allegiance to realist objectivism proves spurious

* Ceci est une version remaniée d'un texte paru en anglais sous le titre de "Reading Althusser" dans Revolutionary World, Vol. 23/25, 1977. Je suis reconnaissant envers Ed Andrew, G.A. Cohen, Frank Cunningham et Phil Hébert pour leur contribution critique au manuscrit. (Le texte fut écrit originellement en 1973-74. Depuis lors, je ne connais aucune occasion où Althusser aurait modifié une position qui lui a été attribuée ici. Il avait en effet déjà abandonné avant 1973 sa position anti-philosophique citée à partir de Lire le Capital dans la note 39 de ce texte, en faveur de la position " non objectiviste " mentionnée à la section 5 ci-dessous. Depuis $1973-74$, il a continué à nous rendre encore plus évidente son adhésion à cette position sur la nature de la philosophie. Dans son avant-propos à Lyssenko : Histoire réelle d'une "science prolétarienne" 'Paris, 1976) de Dominique Lecourt, Althusser relie le phénomène antiscientifique du lyssenkoisme en biologie à " une certaine version, disons ontologique, de la philosophie marxiste " qui « s'interdit de reconnaître que toute la fécondité du matérialisme comme de la dialectique tient à ce qu'ils énoncent non des "lois " mais des thèses . . . (p. 17). Par « lois ontologiques », entendons " la prétendue vérité objective ". Par " thèses ", entendons " jugements de valeur sans prétention de vérité objective ". Du point de vue " ontologique " (objectiviste) rejeté par Althusser, c'est ce dernier qui aurait mis les choses à l'envers et c'est de fait la mise en avant d'une "partisannerie " incompatible avec l'objectivité qui se prête le mieux à susciter des monstruosités politicoscientifiques telles que l'affaire Lyssenko.) La traduction française pour cette édition est par Claude M.J. Braun. 
(even if sincere). In succession, his disapproval of Engels' concept of (objective) relative truth, his distinction between the object of knowledge and the real object, his conception of "ideology", his conception of "empiricism", and his conception of philosophy are examined and criticized.

Dans la mesure où le marxisme vise la scientificité, il tire avantage à mettre à profit toute la rigueur, la limpidité qu'il peut. Par contre, il y a des raisons justifiant une résistance à l'influence théorique de Louis Althusser, - influence fondamentalement antiscientifique et donc antimarxiste - raisons indépendantes de l'indéfendable méthode althussérienne de " lecture symptomale » des textes marxistes. Il suffit de traiter du penchant sans retenue d'Althusser à rendre les questions réellement complexes trop simples (par exemple, l'opposition gnoséologique entre vérité et fausseté, discutée ci-dessous) et son habitude peut-être encore plus ancrée de rendre les questions relativement simples excessivement compliquées (par exemple, il a rebaptisé "surdétermination " la causalité contribuante et il l'invoque pour remplacer la thèse marxiste orthodoxe voulant que l'économique soit la principale cause des grandes lignes de développement historique).

Ma position générale est de contraster défavorablement la position théorique d'Althusser avec celle d'Engels. Cinq thèmes sont traités : 1. La désapprobation éprouvée par Althusser envers la conception engelsienne de la vérité relative 2 . La doctrine d'Althusser voulant que l'objet de connaissance differe nécessairement de l'objet réel 3. La conception d'Althusser de l'idéologie 4. La conception d'Althusser de l'empirisme 5. L'affirmation d'Althusser voulant que la philosopbie n'ait pas d'objet. Je veux aussi fournir des faits indiquant que la réputation d'Althusser pour la rigueur théorique découle beaucoup moins de la sienne propre que du nombre de références qu'il y fait dans ses écrits.

1. Dans sa lettre à Conrad Schmidt datée du 12 mars 1895 , Engels écrivait :

Les objections que vous faites à la loi de la valeur atteignent tous les concepts, à les considérer du point de vue de la réalité. L'identité de la pensée et de l'être, pour reprendre la terminologie hégélienne, coïncide partout avec votre exemple du cercle et du polygone. Ou encore, le concept d'une chose et la réalité de 
celle-ci sont parallèles, comme deux asymptotes qui se rapprochent sans cesse l'une de l'autre sans jamais se rejoindre. Cette différence qui les sépare, c'est précisément celle qui fait que le concept n'est pas d'emblée, immédiatement, la réalité et que la réalité n'est pas immédiatement son propre concept ${ }^{1}$. Du fait qu'un concept possède le caractère essentiel d'un concept, donc qu'il ne coïncide pas d'emblée, prima facie avec la réalité, dont il a fallu d'abord l'abstraire, de ce fait il est toujours plus qu'une simple fiction, à moins que vous n'appeliez fictions tous les résultats de la pensée, parce que la réalité ne correspond à ces résultats que par un long détour et, même alors, ne s'en approche jamais que de manière asymptotique.

\section{À ceci, Althusser répond indigné, au nom de Marx,}

. . . chez Marx en effet le concept de la loi de la valeur est bel et bien un concept adéquat à son objet, puisqu'il est le concept des limites de ses variations, donc le concept adéquat de son champ d'inadéquation, - et nullement un concept inadéquat en vertu d'un péché originel, qui affecterait tous les concepts mis au monde par l'abstraction humaine. Engels reporte donc, comme faiblesse native du concept, sur une théorie empiriste de la connaissance, ce qui fait justement la force théorique du concept adéquat de Marx ! ${ }^{2}$

L'idée d'Althusser, apparemment, est la suivante : l'énoncé scientifique de la loi de la valeur ne fait qu'affirmer l'existence de certaines limites de variation dans la réalité économique ; cette théorie peut donc être entièrement vraie puisque l'élément d'approximation y est déjà explicitement incorporé. Engels préférait

1. En langage coutumier, il y a au moins deux façons d'utiliser le mot " concept " "Begriff " en allemand chez Engels). En parlant, par exemple, du « concept " du système solaire de quelqu'un, nous pouvons nous référer (I) à ce qu'il conçoit être le système solaire, ou (II) à quelque chose de psychologique concernant sa façon particulière de concevoir le système solaire. Puisque (normalement) une personne ne conçoit pas le système solaıre comme étant quelque chose de psychologique en lui, I et II doivent être distincts. Dans ce passage, Engels semble avoir I en tête pour la raison suivante. Ce que nous concevons est marqué d'une imperfection inévitable chez les humains parce que nos descriptions de n'importe quel objet sont incomplètes, ou même incorrectes ; c'est évidemment à cause de cela que ce que nous concevons en concevant, disons, le système solaire, doit nécessairement différer du système solaire tel qu'il existe en réalité ou, en tout cas, être moindre que ce dernier (dans tous ses détails). Tout cela est vrai, malgré le fait que, bien sûr, dans le sens II du mot, aucun " concept " du système solaire, quelle que soit sa perfection, ne pourrait jamais simplement être le système solaire. Pas plus, devrait-on dire, que quelque chose ne puisse jamais être le système solaire, lorsque cette chose ne se trouve que parmi nous qui n'occupons qu'une petite portion du système solaire entier. Par contre, quoique Engels semble en effet avoir utilisé ici le mot " concept " dans le sens I, néanmoins, au début de la section 2 , il semble qu'il ait utilisé le mot dans le sens II.

2. Louis Althusser, Lire Le Capital, Paris, Maspero, 1969, I, 99-100. 
formuler l'énoncé d'une telle loi en termes précis plutôt qu'expressément en termes d'approximation seulement. Pour Engels: un tel énoncé scientifique ne devait pas être absolument vrai mais simplement relativement vrai (chose pourtant objective) comme les conceptions scientifiques en général telles qu'il les a comprises. Cette approche est comprise par Althusser comme étant " empiriste".

De son côté, sans doute Engels appelerait-il l'approche d'Althusser " métaphysique " (c'est-à-dire non dialectique). Ainsi, il énonçait dans Anti-Dübring,

Pour le métaphysicien, les choses et leurs reflets dans la pensée, les concepts, sont des objets d'étude isolés, à considérer l'un après l'autre et l'un sans l'autre, fixes, rigides, donnés une fois pour toutes. Il ne pense que par antithèses sans moyen terme : il dit oui, oui ; non, non ; ce qui va au-delà ne vaut rien. (Chapitre 1, paragraphe 10)

La mentalité métaphysique, disent les engelsiens, est apte à se marier trop intimement avec le mode de pensée caractéristique de la logique formelle où une seule conséquence fausse d'une pensée annule la pensée toute entière. L'approche métaphysique ne connaît que la vérité et la fausseté et n'arrive pas à admettre l'idée d'une chose relativement vraie, c'est-à-dire une théorie plus vraie (objectivement !) que ses rivales sans toutefois être absolument vraie. C'est dans cette ligne de pensée que s'inscrivent les poppériens qui ne peuvent concevoir l'histoire de la science que comme un chemin jonché des os blanchis des théories falsifiées, ne nous donnant aucune raison de supposer que les théories scientifiques courantes ne rencontreront pas à leur tour le même sort, et donc ne laissant aucune raison d'y attacher une confiance dans la pratique ${ }^{3}$. Althusser, par contre, n'a pas de

3. Cf. Engels, Dialectique de la nature, Paris, Éditions sociales, 1968. « Le nombre et la variété des hypothèses qui s'éliminent l'une l'autre, - étant donné le manque de formation logique et dialectique des savants, - donnent alors facilement naissance à l'idée que nous ne pouvons connaître l'essence des choses $"($ p. 243-244). Certains commentateurs ont cru avoir trouvé un lien commun entre le concept dialectique de vérité relative et le concept de Popper de "vérisimilitude ", qu'il explique comme étant la façon selon laquelle une théorie scientifique peut être "plus proche de la vérité " qu'une autre (vọir la thèse de doctorat de Henryk Skolimowsky, à l'Université d'Oxford, 1965, intitulée Polish Analytical Philosophy, p. 446-453 ; cette section a été omise de la version publiée par l'auteur sous le même titre). Popper lui-même maintient énergiquement que son concept donne une réponse à la question à savoir comment la science peut progresser sans jamais être justifiée de se proclamer d'être parvenue à la vérité. Mais on ne trouve pas dans tous les écrits de Popper 
sympathie pour un tel scepticisme complet envers la science et il espère, semble-t-il, assurer à la théorie scientifique la vérité, — c'est-à-dire " la vérité absolue » - en y incorporant dans sa formulation l'approximation explicite partout où elle s'avère nécessaire.

Les poppériens objecteraient que cette stratégie milite contre la falsifiabilité ; et il se peut bien qu'elle prévienne le genre de précision si souvent essentielle pour la mise en pratique autant que pour la vérification des théories scientifiques.

Plus fondamentalement, il semble évident que la stratégie d'Althusser ne peut suffire à lui permettre d'utiliser le titre de vérité scientifique pour la majeure partie de ce que la théorisation scientifique a affirmé dans son histoire jusqu'à aujourd'hui. La physique newtonienne, par exemple, ne peut certainement pas être interprétée comme une approximation explicite de la théorie moderne de la relativité (Au plus, on peut dire qu'elle constitue l'équivalent d'un constat de ce qui se passe selon la physique relativiste sous certaines conditions qui ne sont qu'approximées dans le monde réel.). On ne peut interpréter la géographie supposant une terre plate comme une approximation explicite de la géographie moderne. Ainsi, on doit considérer la physique newtonienne et la géographie supposant une terre plate comme simplement fausses, d'un point de vue métaphysique, puisqu'elles ne sont pas absolument vraies. Est-ce ici la raison pour laquelle Althusser dit que chaque science est fondée historiquement par une " coupure épistémologique » qui n'est pas précédée par quoi que ce soit de scientifique ? L'histoire de la science, bien sûr, ne consiste pas seulement en une série "d'amendements constructifs", pour ainsi dire, aux théories pré-existantes, mais elle contient aussi d'authentiques révolutions scientifiques autant que des périodes d'évolution. L'approche dialectique, par contraste avec celle d'Althusser, est capable d'attribuer une mesure objective et réelle de vérité relative à la théorie

la moindre trace d'une suggestion que son idée de "vérisimilitude " ait été tant sott peu ébauchée par Hegel ou Engels. Peut-être Popper a-t-il raison de souligner sa propre originalité ici parce que son concept semble être très loin du leur. Selon Popper, une fausseté hardie pourrait "s'approcher davantage de la vérité " qu'une proposition absolument vraie la corrigeant. Et assurément, un énoncé soutenant que la température est de 24 degrés devrait être identifié comme plus vrai qu'un énoncé soutenant qu'elle est de 25 degrés, chaque fois qu'elle est en fait de 23 degrés, mais le type de concept poppérien ne donne pas de moyen d'identifier le premier énoncé " s'approchant davantage " de la vérité que le deuxième. 
déposée dans une révolution scientifique aussi bien qu'à son remplacement révolutionnaire. Ce dernier n'est souvent pas du tout une simple négation, mais plutôt un dépassement dialectique, représentant un authentique progrès basé indispensablement sur l'ancienne théorie supplantée. Parce qu'elle conçoit la théorie physique courante, par exemple, comme n'étant elle-même que relativement vraie, la conception dialectique est capable de reconnaître la probabilité d'une future « déposition » révolutionnaire de celle-ci sans lui nier son statut (authentique mais relatif) de connaissance scientifique objective. À défaut du concept dialectique de vérité relative, il semble impossible d'éviter le scepticisme complet quant aux découvertes de la science sans retomber dans un dogmatisme irrationnel en faveur du statu quo dans la science.

2. Commençons cette section comme la précédente, avec une remarque dialectique d'Engels suivie par le reproche d'Althusser (au nom de Marx). Dans sa préface au Volume III du Capital, Engels écrivait :

... du moment où les choses . . . sont conçues . . . comme variables, leurs reflets mentaux, les concepts, sont eux aussi, soumis à la variation et au changement $;. .4$

Le reproche d'Althusser :

Pour pouvoir identifier le développement des concepts et le développement de l'histoire réelle, il faut donc avoir identifié l'objet de la connaissance à l'objet réel, soumis les concepts aux déterminations réelles de l'histoire réelle.

Lorsque Engels dit que nos concepts sont des reflets mentaux des choses, il entend par là qu'ils sont ce qui constitue notre connaissance (ou bien nos fausses conceptions) de ces choses réelles. Il identifie donc l'objet réel avec l'objet de connaissance. Par contre, dans le passage cité plus haut, il ne cherche pas à « identifier le développement des concepts et le développement de l'histoire réelle ». Nous savons de sources indépendantes qu'il considère que le développement de notre connaissance (et de nos fausses conceptions) est une partie du développement de l'histoire réelle. Au lieu d'identifier le développement de la connaissance

4. Karl Marx, Le Capital, Paris, Éditions sociales, 1965, p. 17.

5. L. Althusser, Lire le Capital, I, p. 144. 
d'une part, et le développement de ce-dont-elle-est-laconnaissance d'autre part, Engels nous demande simplement d'accepter qu'il est raisonnable que les deux soient similaires pour ce qui a trait au changement et à la transformation. Après avoir fait référence à ses reproches envers Engels, cités plus haut à la section 1 , Althusser conclut :

Voilà donc jusqu'où porte la méconnaissance de la distinction fondamentale que Marx avait nettement marquée entre l'objet de connaissance et l'objet réel, . . .6

Il est inquiétant de se faire dire que ceci est une distinction si fondamentale. Le lecteur qui connaît la philosophie de John Locke pourra se souvenir ici de sa fameuse définition d'une " idée ": " tout ce qui est l'objet de notre entendement lorsque nous pensons, ... quoi que ce puisse être qui occupe notre esprit lorsqu'il pense ${ }^{7}$. On peut se rappeler avec quelle facilité les berkéléiens ont pu déduire de ceci la conséquence immédiate que, par exemple, ou bien le Mont Blanc est en soi une idée (ou plusieurs idées), ou bien il n'est pas quelque chose à quoi l'on puisse penser ${ }^{8}$. Bien sûr, Locke n'avait pas voulu cette conséquence et Althusser ne la recherche pas non plus. Locke pense que nous obtenons de fait une appréhension empirique, quoique seulement indirecte, des choses du monde extérieur ${ }^{9}$. La façon dont Locke conçoit que nous percevons sensoriellement ces choses a été comparée avec humour à la vision à travers un périscope. On retrouve de ce périscope dans la conception qu'Althusser se fait de la connaissance :

... nous avions vu que le recours à un objet réel primitif ne pouvait nous décharger de penser la différence entre l'objet de la connaissance et l'objet réel, dont le premier objet nous donne la connaissance $;.$. $^{10}$

S'il nous est donné une connaissance de l'objet réel qu'importe le moyen - , il s'ensuit que cet objet devient, lit-

6. Ibid., p. 145 .

7. Essai sur l'entendement humain, Introduction, section 8.

8. Voir, par exemple, le deuxième Dialogue entre Hylas et Philonous, onzième discours de Philonous, de Berkeley : «En dehors des esprits, tout ce que nous connaissons ou concevons, ce sont nos propres idées. Quand donc vous dites que toutes les idées sont occasionnées par des impressions dans le cerveau, concevez-vous ou non, ce cerveau ? . . . " etc.

9. Essai, Livre IV, Chapitre IV, section 3: "Il est évident que l'esprit ne connaît pas les choses immédiatement, mais seulement par l'intervention des idées qu'il en a ; "

10. Op. cit., p. 78. 
téralement, un objet de connaissance pour nous. Puisque nous en étions à une analyse littérale d'Althusser, il serait peut-être approprié maintenant de tenter une lecture non littérale du fameux diction d'Althusser selon lequel « l'objet de connaissance » est différent de l'objet réel.

... il ne faut pas confondre les deux objets : l'idée du cercle, qui est l'objet de la connaissance, avec le cercle, qui est l'objet réel. ${ }^{11}$

Nous avons pourtant assez avancé ce travail pour pouvoir aborder, en revenant à la différence d'ordre entre l'objet de la connaissance et l'objet réel, le problème dont cette différence est l'indice : le problème du rapport entre ces deux objets (objet de la connaissance et objet réel), rapport qui constitue l'existence même de la connaissance. ${ }^{12}$

. . . le processus de production d'une connaissance passe nécessairement par la transformation incessante de son objet (conceptuel) ; . . . cette transformation, qui fait un avec l'histoire de la connaissance, a justement pour effet de produire une nouvelle connaissance (un nouvel objet de connaissance), qui concerne toujours l'abjet réel, dont la connaissance s'approfondit justement par le remaniement de l'objet de connaissance. ${ }^{13}$

Ces brèves citations, comme d'autres passages de ses écrits, portent à interpréter "l'objet de connaissance " d'Althusser comme «l'objet-tel-que-connu ». Ainsi, l'objet de la connaissance géométrique du cercle serait non pas le cercle, mais le cercle-tel-que-connu-géométriquement, c'est-à-dire dans le cas de la géométrie euclidienne, par exemple, le cercle euclidien pourtant il faut dire que strictement parlant, ceci n'est que le cercle prétendument connu en géométrie euclidienne (puisque la géométrie riemanienne est plus vraie, pense-t-on maintenant). De même, selon notre présente interprétation, «l'objet " de la physique newtonienne serait la-réalité-physique-telle-quedécrite-par-la-physique-newtonienne. De la même façon, on attribuerait au dicton d'Althusser voulant que "l'objet de connaissance " soit toujours différent de l'objet réel, la portée de signaler qu'on ne pourra jamais s'attendre à ce que les théories scientifiques significatives soient absolument (c'est-à-dire entiè-

11. Ibid., p. 46.

12. Ibid., p. 61.

13. Ibid., p. 20. 
rement) vraies puisqu'elles ne consistent que d'abstractions humaines, tandis que la réalité, c'est la réalité. Malheureusement, c'est justement cet énoncé d'Engels qu'Althusser a réprouvé si sévèrement en dénonçant les conséquences du fait d'ignorer la « distinction fondamentale . . . entre l'objet de connaissance et l'objet réel »! Nous devrions alors chercher une autre interprétation de ce qu'entend Althusser par l'expression « objet de connaissance ${ }^{14}$,

De fait, il existe un texte où Althusser énonce expressément ce qu'il entend par " objet de connaissance " « au sens rigoureux du terme ».

. . . il y a loin par exemple entre la matière première sur laquelle travaille Aristote et la matière première sur laquelle travaillent

14. J'ai reçu une communication personnelle où l'on raisonne que la lecture d'Althusser proposée dans ce paragraphe n'arrive pas à réduire sa position à celle d'Engels citée plus haut, mais à quelque chose de plutôt banal.

"Supposons que je décris ma plume comme étant bleue et mesurant 4 pouces de longueur. Je n'en dis pas plus. Il s'ensuit que ma-plume-telle-que-décrite-par-moi est bleue et mesure 4 pouces, mais n'est pas faite d'acier, alors que ma plume, elle, l'est. Ainsi nous avons une différence entre ma plume telle que je la décris et ma plume. Mais ceci ne veut pas dire que ma description de ma plume n'est pas absolument vraie. Ainsi le rejet de la catégorie de vérité absolue se trouve ailleurs que dans la distinction entre les concepts et la réalité si celle-ci est une distinction entre ca-tel-que-décrit-par-p) et (a) ".

De fait, il n'est pas difficile d'apprécier l'idée de l'auteur de cette objection quand il affirme une différence entre sa plume et sa-plume-telle-que-décrite-par-lui. Mais ne semble-t-il pas étrange de décrire cette dernière comme n'étant définitivement " pas faites d'acier " ? Le terme " indéterminé " ne serait-il pas plus précis pour décrire ce dont est constituée sa-plume-telle-que-décrite-par-lui ? Ainsi, la différence entre la plume de l'objecteur et sa-plume-telle-que-décrite-par-lui ne serait une différence que de richesse qualitative, pour ainsi dire. Cette interprétation indiquerait que l'insistance d'Althusser à distinguer «l'objet de connaissance " de l'objet réel ne serait qu'une façon d'insister que nous ne puissions être omniscients de quoi que ce soit. Il n'y aurait pas nécessairement d'implication d'une quelconque fausseté dans notre " connaissance ", mais seulement qu'elle est à court de vérité ; c'est-à-dire que la seule idée sur laquelle Althusser insiste ici est la reconnaissance du fait que notre connaissance des choses doit nécessairement toujours être incomplète, peu importe qu'elle soit dépourvue de fausseté dans ce qu'elle affirme. Selon cette lecture, la distinction d'Althusser entre "l'objet de connaissance " et l'objet réel consisterait simplement en une distinction entre ce-que-nous-connaissons d'une chose et ce-qui-estconnaissable (- ou inconnaissable - ), ce dont le connu ne formerait qu'une partie. Toutefois, une telle interprétation de l'intention d'Althusser semble contredire le fait que plus haut dans le même livre, Lire le Capital, Tome I., il avait, avec une extraordinaire véhémence, reproché d'être empiriste, à la doctrine suivante : "La connaissance (qui n'est que l'essence essentielle) est contenue réellement dans le téel comme une de ses parties, . . . ( (p. 40) ; et deux pages plus loin, Althusser identifiait " l'essence de l'objet réel " selon le point de vue de "l'empirisme " en formulant de la façon suivante une thèse sur le réel qui, d'après lui, définit l'empirisme : " La connaissance y est tout entière réellement présente : non seulement son objet, qui est cette partie réelle appelée essence, mais aussi son opération ... " (p. 42). (Une discussion critique plus étendue de la conception althussérienne de "l'empirisme " se trouve plus loin à la section 4.) 
Galilée, Newton ou Einstein, - mais . . formellement cette matière première fait partie des conditions de la production de toute connaissance . . . s'il est clair à chacun que cette matière première devient, au fur et à mesure que progresse une branche de la connaissance, de plus en plus élaborée, si la matière première d'une science développée n'a évidemment plus rien à voir avec la "pure " intuition sensible ou la simple "représentation ", en revanche aussi loin qu'on remonte dans le passé d'une branche de la connaissance, on n'a jamais affaire à une intuition sensible, ou représentation "pures ", mais à une matière première toujours-déjà complexe, à une structure d' "intuition " ou de " représentation » combinant, dans une "Verbindung » propre, à la fois des « éléments » sensibles, des éléments techniques, et des éléments idéologiques ; . . donc jamais la connaissance ne se trouve, comme le voudrait désespérément l'empirisme, devant un objet pur qui serait alors identique à l'objet réel dont la connaissance vise justement à produire . . . la connaissance. La connaissance travaillant sur son " objet ", ne travaille pas alors sur l'objet réel, mais sur sa propre matière première, qui constitue, au sens rigoureux du terme, son "objet " (de connaissance), qui est, dès les formes les plus rudimentaires de la connaissance, distinct de l'objet réel,... . 15

Malgré ce que "voudrait désespérément l'empirisme ", dans la conception d'Althusser, certains lecteurs se souviendront des philosophes (soit Quine, Popper, et Feyerabend) qui, tout en s'appelant " empiristes », nient catégoriquement la possibilité de découvrir un "donné » expérientiel pur qui soit neutre du point de vue théorique. De toute façon, dans ce passage, Althusser fait travailler un de ses thèmes préférés, selon lequel le processus de "production de la connaissance » est un processus de production avec ses propres «matières premières » et «produits $"{ }^{16}$. (En particulier, il s'agit du processus de production des «Généralités III " à partir des «Généralités I » avec l'aide instrumentale des «Généralités II » d'ordre méthodologiquethéorique.) Ici Althusser semble dire que les « objets » de connaissance scientifique sont les "matières premières " dans le processus de production de la connaissance ou, en d'autres termes,

15. Ibid., p. 49.

16. Le processus de production scientifique est présenté comme une " pratique théorique "; seulement on remarquera l'énoncé d'Althusser concernant les " sciences rexpérimentales) " disant que " leurs expériences . . . constituent la forme de leur pratique théorique " (Lire le Capital, p. 72). Ainsi, la " pratique théorique " d'Althusser a un sens plus large, plus inclusif, que ne l'a ordinairement le mot « théorisation». 
qu'ils sont les données - pourtant non pas ultimes et absolues mais produites plutôt par des processus de production théorique antécédents. Il reste qu'Althusser doit entendre par ces « matières premières " de la théorisation de deux choses l'une : soit les ensembles de connaissances-erreurs avec lesquels tout effort de théorisation doit commencer (du moins dans l'instance immédiate); soit des choses (putatives) que chaque ensemble de connaissances-erreurs prétend décrire correctement. Dans le premier cas, il est difficile de voir comment il a pu penser qu'Engels ait confondu "l'objet de connaissance " avec les objets réels puisque ce dernier a toujours fait une distinction très nette, dans les passages cités ici et partout dans sa philosophie, entre les " choses " d'un côté, et leurs "reflets dans la pensée, les concepts", de l'autre ${ }^{17}$. Dans le deuxième cas, «l'objet " de la physique newtonienne, par exemple, n'aurait pas consisté en la réalité physique telle que décrite par Newton, mais plutôt en la réalité telle que décrite par les préconceptions avec lesquelles Newton a dû commencer son activité théorique (c'est-à-dire sa "pratique théorique »). Dans ce cas, la force de l'affirmation que « l'objet de connaissance » n'est pas l'objet réel se limiterait à dire que les préconceptions ne peuvent être absolument correctes. Par contre, si nous disons que les "Généralités III ", c'est-à-dire les «postconceptions » théoriques, les résultats (temporairement) finaux de la théorisation scientifique, ne sont jamais absolument corrects, alors, selon Althusser, nous tombons avec Engels dans «l'empirisme ». Mais le problème ici est que ce qui sera interprété comme préconception ou " matière première » à un stade donné du processus de "production » théorique aura été perçu comme postconception ou " produit fini " par rapport à un stade antérieur du processus. "L'objet » de connaissance, nous assure Althusser, « est, dès les formes les plus rudimentaires de la connaissance, distinct de l'objet réel ». Mais nous voulons une réponse à la question suivante : est-ce que cette distinction tient pour les plus hautes formes de connaissance? Althusser ne peut admettre que ces dernières n'at-

17. En effet, dans son livre Lénine et la philosophie, Paris, Maspero, 1969, p. 39, Althusser mentionne expressément la " catégorie de reflet " comme étant, chez Lénine, une des " catégories contaminées par ses références empiristes " dans Matérialisme et empiriocriticisme. 
teignent la vérité absolue (sous peine de tomber dans « l'empirisme " engelsien). Peut-être pourrait-il dire que toutes les préconceptions théoriques sont incorrectes, mais pas toutes les postconceptions théoriques. Il pourrait aussi dire que lorsque le " processus de production » théorique arrive à générer un " produit fini » possédant la vérité absolue, le processus de production théorique est achevé et ce "produit fini » ne servira jamais comme « matière première » pour un processus productif subséquent - (car s'il le faisait, cela produirait un exemple d'un objet réel qui est en même temps un " objet de connaissance »). Dans ce dernier cas, la thèse d'Althusser voulant que « l'objet de connaissance » differe toujours de l'objet réel signifierait que tout processus de théorisation scientifique procède jusqu'à l'atteinte de la vérité absolue où il s'arrête pour toujours. On doute sérieusement que cette interprétation corresponde à l'intention d'Althusser.

3. Abordons cette section avec une question. Il s'agit d'une question posée par Norman Geras, un admirateur critique d'Althusser, à la conclusion de son utile article synoptique, intitulé "Althusser's Marxism : An Account and Assessment" dans New Left Review, No 71, janvier-février 1972. La discussion de Geras, quoiqu'en grande partie admirative, contient quelques commentaires sévères, dont le plus âpre accuse Althusser d'idéalisme et d'élitisme.

. . la connaissance apportée par le marxisme et importée par les intellectuels dans le mouvement ouvrier, joue, d'après lui, un rôle directeur très spécifique. Elle tente de produire «dans les masses une nouvelle forme d'idéologie [omis par Geras : « (une idéologie qui repose, cette fois, sur une science, - ce qui ne s'était jamais vu) " Lire le Capital, Tome I, page 166.]) . . . Mais Althusser nous dit aussi, dans une centaine de passages au moins, que l'idéologie est un domaine de mystification et déformation, d'illusion, fausseté et mythe, de confusion, préjudice et arbitraire, d'imaginaire et non-connaissance [une note au bas de la page ici donne vingt-trois citations à l'appui]. Il sépare ainsi les masses de la connaissance de leur situation, produite par les intellectuels, par une nécessité qu'il n'explique jamais. Comment les intellectuels peuvent-ils alors brandir ce qu'ils savent être une idéologie sans violer le principe premier de la politique révolutionnaire, d'affirmer les faits tels qu'ils sont? (page 86) 
De fait, Althusser nous donne une réponse à cette question, mais pas celle à laquelle on aurait pu s'attendre. En tout cas, c'est une réponse propre à surprendre les lecteurs éduqués dans certaines traditions philosophiques.

Pour résoudre cette énigme, il faudra s'interroger sur la signification donnée par Althusser au terme "idéologie ». Malheureusement le terme n'est pas du tout univoque. Voici sept différents sens trouvés dans les écrits marxistes et non-marxistes, - dont aucun, tâcherai-je de montrer, ne correspond à l'usage d'Althusser : (a) science des idées (la signification originelle datant du dix-huitième siècle et maintenant désuète) ; (b) la surconcentration sur la théorisation et les idées (également désuète) ${ }^{18}$; (c) la composante non-économique de l'ordre social (par exemple, art, politique, jurisprudence, religion, philosophie) ${ }^{19}$; (d) un corpus d'idées redevable de sa popularité à sa fonction, - sans que ses adhérents ne s'en rendent compte, - de glorifier une des spécialités occupationnelles non-économiques créées par la division du travail, tout en exagérant l'autonomie relative de (l'objet traité par) cette spécialité pour ainsi obnubiler le fait que ses grandes lignes soient vraiment déterminées à la longue par l'économique ; (e) système d'idées partisan et inacceptable du point de vue scientifique ; $(f)$ système d'idées partisan et " non-objectif » (c'est-à-dire ni vrai, ni faux) ; $(\mathrm{g})$ système partisan d'idées. Je présume qu'aucune de ces interprétations du mot "idéologie ", sauf peut-être (c), n'apparaîtra nouvelle au lecteur. De ces sept différents sens, $(\mathrm{g})$ est le plus communément utilisé, même parmi les marxistes orthodoxes ; (f) est répandu

18. Pour une discussion de ces deux sens, voir l'essai de George Lichteim, "The Concept of Ideology", dans son livre The Concept of Ideology and Other Essays, New York, Random House, 1967.

19. «Le changement dans la base économique bouleverse plus ou moins rapidement toute l'énorme superstructure. Lorsqu'on considère de tels bouleversements, il faut toujours distinguer entre le bouleversement matériel - qu'on peut constater d'une manière scientifiquement rigoureuse - des conditions de production économiques et les formes juridiques, politiques, religieuses, artistiques ou philosophiques, bref, les formes idéologiques sous lesquelles les hommes prennent conscience de ce conflit et le mènent jusqu'au bout " (Karl Marx, Préface de la Contribution à la critique de l'économie politique, Paris, Éditions sociales, 1972, p. 4-5). Ma suggestion est que le mot "idéologie » se réfere ici à tout le domaine et seulement le domaine de la "superstructure ". Lénine commentait ce passage en disant que chez Marx et Engels, l' « idée fondamentale . . était que les rapports sociaux se divisent en rapports matériels et rapports idéologiques. Ces derniers ne sont qu'une superstructure érigée sur les premiers ... ( (Oeuvres Complètes, Tome I, Paris, Éditions sociales-progrès, 1977, paragraphe 33). 
parmi ceux qui sont le plus influencés (d'après les engelsiens) par la thèse philosophique bourgeoise, - autant positiviste qu'existentialiste, - voulant que la «partisannerie » soit incompatible avec l'objectivité ; (d) bien sûr, est l'interprétation la plus fréquente de Marx et Engels, par exemple dans l'Idéologie allemande.

Mais je crois qu'aucune de ces sept significations ne correspond à l'usage d'Althusser. Dans sa préface spéciale "To My English Readers", dans For Marx (Pour Marx) ${ }^{20}$, Althusser commente son essai de 1963 " Marxisme et humanisme " (chapitre 7 de For Marx) :

Je ne condamnais en aucune façon l'idéologie en tant que réalité sociale : comme le disait Marx, c'est dans l'idéologie que les hommes sprennent conscience) de leur conflit de classe et «le mènent jusqu'au bouts. Dans ses formes religieuses, éthiques, légales et politiques, etc. , l'idéologie est une réalité sociale objective ; la lutte idéologique fait partie organiquement de la lutte des classes. D'un autre côté, j'ai critiqué les effets théoriques de l'idéologie, qui sont toujours une menace ou entrave à la connaissance scientifique. (p. 11-12)

Althusser dit que l'idéologie au niveau de ses effets théoriques est toujours antiscientifique. Qu'adviendrait-il si nous demandions à Althusser, comme Geras : pourquoi les hommes, du moins de nos jours, ne peuvent-ils pas prendre conscience de leur conflit de classe par la science marxiste plutôt que par l'idéologie? Pourquoi l'idéologie doit-elle, même pour les marxistes, faire toujours «partie organiquement de la lutte des classes »? Une première réponse que l'on peut imaginer représentative de la position d'Althusser est que la science marxiste dépasse les capacités intellectuelles des masses. Il faut bien admettre que les concepts scientifiques marxistes doivent se faire simplifier dans le processus de vulgarisation et devenir alors quelque peu grossiers. Mais le marxisme orthodoxe nierait que les conceptions des masses, lorsqu'elles embrassent le marxisme, doivent prendre pour cette raison une forme par nécessité essentiellement non-scientifique ; selon le point de vue du marxisme orthodoxe, ces conceptions sont toujours capables d'accéder à la vérité relative même si elles n'atteignent pas pleinement la scientificité. Selon

20. Londres, Allen Lane The Penguin Press, 1969. 
l'opinion d'Althusser toutefois (un « métaphysicien " profondément ancré dans le sens du mot tel qu'utilisé par Engels), ces conceptions doivent constituer une "idéologie " (quoiqu'une " idéologie qui repose sur une science »), et une idéologie ne peut jamais contenir quoi que ce soit d'authentiquement scientifique.

Toutefois, quant à la proposition d'Althusser niant que la science marxiste peut remplacer l'idéologie dans la lutte de classe prolétarienne, il y a de bonnes raisons de conclure qu'elle ne se base sur aucune limitation intellectuelle des masses. Car cela mènerait à une conception élitiste où les savants marxistes dans un mouvement ou état socialiste joueraient un rôle similaire aux " gardiens" de la République de Platon, étant les seuls à connaître la vérité, et régimentant les masses par de "beaux mensonges ", la mythologie délibérément inventée pour sanctifier tout l'arrangement social. À la page 242 de Pour Marx, Althusser rejette expressément cette conception de l'idéologie en disant qu'elle n'est pas une machination délibérée et que la classe dirigeante dans toute société de classe y est autant assujettie que les classes dirigées et qu'aucune société (même communiste) ne peut y échapper.

.. . l'idéologie (comme système de représentations de masse) est indispensable à toute société pour former les hommes, les transformer et les mettre en état de répondre aux exigences de leurs conditions d'existence . . . C'est dans l'idéologie que la société sans classe vit l'inadéquation-adéquation de son rapport au monde, en elle et par elle qu'elle transforme la " conscience " des hommes, c'est-àdire leur attitude et leur conduite, pour les mettre au niveau de leurs tâches et de leurs conditions d'existence.

Ici il semble qu'Althusser conçoit l'idéologie comme socialement indispensable parce que l'inspiration et la consolation personnelles sont (évidemment) socialement indispensables dans quelque société que ce soit. À la lumière de ceci, nous sommes en meilleure position pour comprendre l'énoncé d'Althusser deux pages plut tôt :

Dans l'idéologie, le rapport réel est inévitablement investi dans le rapport imaginaire : rapport qui exprime plus une volonté . . . qu'il ne décrit une réalité. 
Ici on peut se croire en terrain familier. L'objectiviste éthique (comme l'engelsien ${ }^{21}$ ) voudra y objecter qu'en effet, certains jugements de valeurs sont objectivement vrais alors que d'autres ne le sont pas. Mais de toute façon, la position citée plus haut semble en être une qu'on rencontre assez souvent. Il reste pourtant l'énigme suivante : pourquoi Althusser maintient-il avec insistance que les jugements de valeur, éthiques et autres - pour la seule raison qu'ils ne peuvent être objectifs (vrais) - devront toujours représenter « une menace ou entrave à la connaissance scientifique "? Ce que croit vraiment Althusser est qu'il y a quelque chose d'essentiellement anti-scientifique, et non pas seulement non-scientifique au domaine des valeurs. Où avonsnous donc déjà été témoin de ce point de vue?

Dans son essai « Idéologie et appareils idéologiques d'état », Althusser écrit :

. . . la catégorie de sujet est constitutive de toute idéologie, . . .

. . . il faut être averti que aussi bien celui qui écrit ces lignes, que le lecteur qui les lit, sont eux-mêmes des sujets, donc des sujets idéologiques (proposition tautologique), c'est-à-dire que l'auteur comme le lecteur de ces lignes vivent "spontanément " ou « naturellement » dans l'idéologie, au sens où nous avons dit que « l'homme est par nature un animal idéologique».

. . . tout discours scientifique est par définition un discours sans sujet. $^{22}$

Ainsi, la raison pour laquelle " l'idéologie » et la science s'opposent inévitablement, selon Althusser, est que l'idéologie est constituée de la «catégorie de sujet » alors que la science est " sans sujet". Nous n'avons besoin maintenant que de comprendre ce qu'Althusser croit être la « catégorie de sujet » pour résoudre la présente énigme de la compréhension de la théorie althussérienne.

Dans l'acceptation courante du terme, sujet signifie en effet 1) une subjectivité libre : un centre d'initiatives, auteur et responsable de ses actes ; 2) un être assujetti, soumis à une autorité

21. "Qu'avec cela, il se soit en gros effectué un progrès, pour la morale comme pour toutes les autres branches de la connaissance humaine, on n'en doute pas ». Anti-Dübring, Paris, Éditions sociales, 1963, p. 126.

22. L. Althusser, Positions, Paris, Éditions sociales, 1976, p. 110-111. 
supérieure, donc dénué de toute liberté, sauf d'accepter librement sa soumission. ${ }^{23}$

Interprétation : un « sujet » est 1) un être libre et responsable ; et 2) un être qui souscrit (se soumet librement) à un système de valeurs en le reconnaissant comme autorité.

.. . nous constatons . . . que la représentation idéologique de l'idéologie est elle-même contrainte de reconnaître que tout « sujet », doté d'une " conscience », et croyant aux "idées » que sa " conscience " lui inspire et accepte librement, doit « agir selon ses idées », doit donc inscrire dans les actes de sa pratique matérielle ses propres idées de sujet libre. S’il ne le fait pas, «ce n'est pas bien $"{ }^{24}$

Althusser croit, apparemment, que pour vivre nous avons besoin de nous concevoir (et de concevoir les autres) comme des agents humains libres, responsables de nos actes, alors que dans la science, de telles conceptions doivent "par définition " être écartées comme illusoires. C'est Emmanuel Kant qui, le premier, a développé la doctrine de l'homme qui habite "deux mondes ", un monde de " raison pratique » où l'homme en tant qu'agent pratique se conçoit nécessairement comme libre et responsable, et un monde de "raison théorique ", où l'homme en tant que scientifique doit forcément concevoir tout ce qui se passe comme totalement prédéterminé causalement, et donc sans aucune liberté ou aptitude à être tenu responsable. (Quant au point de vue d'Engels ainsi que de plusieurs autres philosophes datant au moins du seizième siècle jusqu'à aujourd'hui, — selon lequel le déterminisme causal total est néanmoins compatible avec la liberté humaine et la responsabilité morale telles que couramment comprises, - cette notion ne semble pas avoir traversé l'esprit ni de Kant ni d'Althusser, car nous ne trouvons aucune tentative de réfutation ni chez l'un ni chez l'autre.) Nous sommes maintenant bien placé pour comprendre ce qu'entend Althusser par "l'anti-humanisme théorique " qu'il considère essentiel pour la science : il faut lire ici le fatalisme théorique.

Voici une objection simple à toute tentative de soutenir une quelconque idée kantienne des " deux mondes », épousée,

23. Ibid., p. 121.

24. Ibid., p. 107. 
soit par Althusser, soit par quelqu'un d'autre. Il n'y a que deux possibilités. Althusser croit à l'existence d'au moins une mesure de liberté humaine ou il n'y croit $\mathrm{pas}^{25}$. S'il y croit, il doit admettre qu'à son avis la science ne peut la nier (elle ne peut exclure la " catégorie du sujet ") qu'en tombant dans l'erreur, et alors, ce n'est pas après tout l'idéologie, mais la science, qui est sujette à l'illusion et à la non-connaissance. Par contre, s'il ne croit pas vraiment à l'existence de la liberté humaine, alors lui-même n'est pas de fait un " animal idéologique ", et ainsi l'idéologie doit être une condition psychologiquement guérissable. On dirait quasiment que Lénine répondait à Althusser plutôt qu'à Ernst Mach lorsqu'il écrivait dans Matérialisme et empiriocriticisme :

Borner le déterminisme au domaine des " recherches " et laisser, en morale, dans la vie sociale, dans tous les autres domaines, la question à l'appréciation « subjective ", n'est-ce pas faire preuve d'obscurantisme? «Dans mon cabinet, dit le pédantesque savant, je suis déterministe "; mais il ne souffle mot du devoir du philosophe de bâtir sur le déterminisme un système complet embrassant la théorie et la pratique. ${ }^{26}$

4. Bien sûr, le Matérialisme et empiriocriticisme de Lénine, nous dit Althusser ${ }^{27}$, est jusqu'à un certain point, gâté par « l'empirisme» (aussi parce qu'il est pré-kantien ${ }^{28}$ ). Mais quel est donc cet « isme » qu'Althusser appelle « empirisme » ? Quels en sont les fondements?

Pour voir jusqu'à quel point Althusser fait une utilisation compréhensive du mot " empirisme », considérons sa déclaration suivante :

Qu'un objet ne puisse être défini par son apparence immédiatement visible ou sensible, qu'il faille passer par le détour de son concept pour le saisir . . . voilà une thèse qui résonne à nos oreilles comme quelque chose de déjà connu : c'est du moins la leçon de toute l'histoire de la science moderne, plus ou moins

25. Il est possible, bien sûr, d'avoir des croyances contradictoires, mais sûrement pas d'adopter et rejeter simultanément la même croyance. Il est sûrement impossible de dire d'une personne qu'elle adopte une " croyance " si elle n'est pas, dans l'ensemble, psychologiquement plus persuadée de la proposition dont il s'agit que de sa négation.

26. Vladimir Illich Lénine, Matérialisme et empiriocriticisme, Paris, Éditions sociales, 1951, p. 170 .

27. Althusser, Lénine et la philosophie, p. 38.

28. Ibid., p. 34, 38 . 
réfléchie dans la philosophie classique, même si cette réflexion s'est opérée dans l'élément d'un empirisme transcendant (comme chez Decartes), ou transcendental (Kant et Husserl) ou idéaliste « objectif » (Hegel). (. . .) la connaissance d'un objet réel passe non pas par le contact immédiat avec le " concret " mais par la production du concept de cet objet (au sens d'objet de connaissance), comme par sa condition de possibilité théorique absolue. ${ }^{29}$

Quelle est cette « leçon de toute l'histoire de la science moderne " qu'Althusser veut nous rappeler? En tirant ces quelques mots du passage précédent :

. . . un objet ne [peut] être défini par son apparence immédiatement visible ou sensible, . . . il [faut] passer par le détour de son concept pour le saisir . . . la connaissance d'un objet réel passe non pas par le contact immédiat avec le " concret " mais par la production du concept de cet objet....,

nous semblons arriver à une réponse. Althusser semble vouloir nous rappeler ce truisme familier mais crucial de la dialectique : les premières impressions de quelque chose sont souvent illusoires et toujours incomplètes; une conception plus profonde de la chose s'approchant davantage de la vérité requiert un travail de la raison. Dénommons ce truisme « $\mathrm{P} »$. Mais si l'idée d'Althusser ne va pas plus loin que cela, pourquoi introduit-il ici "l'objet de connaissance ", et en quoi, précisément, les philosophies de Descartes, Kant, Husserl et Hegel sont-elles pertinentes? Parmi ces quatre philosophes, ce n'est qu'à Hegel qu'on peut attribuer un intérêt à l'égard de la thèse $\mathrm{P}$ (et même Hegel se serait refusé de qualifier de "détour " la progression de l'expérience sensible vers le concept rationnel). Quant à Husserl, il est probablement préférable de l'interpréter comme s'opposant à $\mathrm{P}$. La sympathie du philosophe Descartes et probablement aussi de Husserl, du moins dans ses débuts, pour la « théorie périscopique de la perception indirecte " mentionnée plus haut en connection avec Locke, semble pertinente à ce qu'Althusser essaie d'avancer. Althusser tente-t-il de nous dire que notre façon de saisir un objet par la production d'un concept est de saisir plutôt le concept ? C'est-à-dire, en réalité, en ne saisissant pas l'objet ? La philosophie de Kant concernant la chose qui est l'objet de connaissance affirmait que cette dernière était

29. Althusser, Lire le Capital, II, p. 59. 
un produit de la pensée conceptuelle et qu'elle devait nécessairement différer de la chose telle quelle en soi. Or Althusser n'essaie sûrement pas de nous présenter une pareille doctrine!

Mais ceci était une digression. Notre problème est de spécifier des fondements de cet « empirisme » contre lequel Althusser lutte si farouchement :

(i) le réel c'est ce qui est immédiatement discernable par l'expérience sensorielle sans l'aide de la pensée conceptuelle ${ }^{30}$;

(ii) la connaissance du réel est obtenue par l'abstraction d'une « essence » qui s'y trouve ${ }^{31}$;

(iii) la connaissance d'une chose réelle consiste à saisir son essence qui est cachée par les apparences immédiates ${ }^{32}$;

(iv) la connaissance d'un objet réel fait elle-même partie de cet objet à connaître ${ }^{33}$;

(v) la connaissance constitue un "modèle » de la réalité ; mais la réalité elle-même est " toujours-plus-riche-et-plusvivante-que-la-théorie " ${ }^{34}$.

De ces cinq thèses, seulement (i) et probablement (iv) seront normalement qualifiées d' " empiristes » dans toute l'acception courante du terme. La thèse (i) est pratiquement une négation directe de la thèse $P$, et c'est nettement (i) que, par exemple, Marx et Engels entendaient par empirisme dans L'Idéologie allemande (Paris, Éditions sociales, 1971), p. 51.

La thèse (iv), prise littéralement, a été, de fait, réitérée par des empiristes « monistes neutres ", tel Ernst Mach qui identifie la connaissance avec les rapports fonctionnels entre les phénomènes de l'expérience en refusant expressément de faire une distinction ultime entre les rapports eux-mêmes et l'appréhension mentale que nous en avons. Mais il est peu probable qu'Althusser ait eu le « monisme neutre » en tête ici puisqu'il ne le mentionne pas du tout. Il déduit plutôt la soumission de "l'empirisme » à la thèse (iv) à partir de la conception «empiriste " des essences. Selon Althusser, puisque «l'empirisme » soutient que la con-

\footnotetext{
30. Ibid., II, p. 57-58.

31. Ibid., I, p. 40-41.

32. Ibid., I, p. 41.

33. Ibid., I, p. 42.

34. Ibid., I, p. 148.
} 
naissance d'une chose réelle est la connaissance de son essence et admet aussi qu'il y a néanmoins plus dans une chose réelle que ce qui est contenu dans son essence, il s'ensuit que "l'empirisme " est obligé de concevoir le réel comme étant divisé en deux parties et d'identifier l'une de ces parties avec la connaissance du réel ${ }^{35}$. Or, Althusser ne veut sûrement pas dire que "l'empirisme " raisonne ainsi consciemment à partir de son concept d'essences jusqu'à la thèse (iv), car nulle part dans ses écrits ne mentionne-t-il que quiconque, avant lui, empiriste avoué ou non, ait soufflé mot d'une telle inférence. Il faut donc qu'il considère cette dernière comme valide et que l' " empiriste " soit soumis à la thèse (iv), qu'il le veuille ou non, en vertu de sa conception de la connaissance comme fondée sur des essences. Mais ceci est une erreur. De fait, interpréter la connaissance d'une chose réelle comme la connaissance de son essence implique la division, pour ainsi dire, de cette chose en deux, en ce qui y est essentiel et ce qui n'y est pas essentiel. Mais pourquoi l'essentiel doit-il être conçu comme étant la même chose que la connaissance qu'on en a ? Le terme " connaissance " est sans doute ambigu. Il peut indiquer soit ce qui est connu (c'est-à-dire un ensemble de faits), soit l'état mental par lequel on les connaît. On ne peut considérer ces faits que comme existant dans la réalité en dehors de notre pensée, et on ne peut considérer cet état mental que comme produit de la pensée. Althusser semble penser, par exemple, que si une personne considère (avec assez de bon sens) que ce qui est connu actuellement en botanique comprend une partie seulement de tout ce qui existe en réalité dans le domaine de la botanique, cette personne doit être rationnellement tenue d'admettre que l'état mental par lequel nous connaissons les faits botaniques fait d'une quelconque façon partie de tout ce qui existe en réalité en botanique. Mais notre connaissance des faits botaniques n'est évidemment pas un fait botanique. Cette interprétation du raisonnement d'Althusser peut paraître hirsute au point de la mettre en doute. Mais quelle autre interprétation ses énoncés permettent-ils?

Il ne sera pas nécessaire de discuter longuement la thèse (v) de "l'empirisme " puisqu'il semble s'agir à peu de choses

35. Ibid., 1, p. 42. 
près de la doctrine engelsienne exprimée dans la lettre à Schmidt, doctrine qu'Althusser a attaquée dans un passage cité plus haut. Althusser ajoute dans ce même contexte que si l'on conçoit la connaissance comme un "modèle " de quelque chose qui est " toujours-plus-riche-et-plus-vivant-que-la-théorie », on doit finir par épouser le pragmatisme.

Cette opération a pour effet inévitable de faire penser la théorie comme un instrument empirique, parmi d'autres, bref de réduire directement toute théorie de la connaissance comme modèle à ce qu'elle est : une forme de pragmatisme théorique. ${ }^{36}$

Une fois de plus, nous voyons le prix que doit payer Althusser pour avoir rejeté le concept engelsien de vérité relative qui, dans la conception d'Engels, bien sûr, est néanmoins objectif exactement dans le sens déploré par le pragmatisme philosophique. Pour Althusser, si une théorie ne peut pas être conçue comme absolument vraie, elle doit "inévitablement " être conçue comme un simple "instrument empirique parmi d'autres».

Considérons maintenant quelques-unes de ces thèses " empiristes " dans leurs rapports les unes aux autres. La thèse (i) dit que la réalité est tout, et rien que, ce qui est appréhendé dans l'expérience sensorielle immédiate. La thèse (ii) dit que la connaissance ne s'occupe que d'une partie du réel. La thèse (iii) attribue à la connaissance la propriété de contredire l'expérience sensorielle immédiate (quelle autre force pourrait-on donner à l'idée de l'essence cachée par les apparences immédiates ?). Comment est-il possible qu'une personne adopte toutes ces thèses? Oui, en étant inconséquent, dira-t-on. Mais se peut-il qu'un théoricien ait été si inconséquent? On s'attend à ce que quiconque accepte (i) rejette (iii) et que quiconque accepte (iii) rejette (i). Mais il est peut-être possible de nommer au moins un penseur qui est assez embrouillé pour se contredire à un tel point, - en l'occurrence, Althusser. Puisqu'il rejette (i), il semble qu'Althusser lui-même est tenu d'adopter soit la thèse (ii), soit (iii) malgré toute sa véhémence contre la " catégorie de l'essence ».Car en adoptant la " catégorie de l'essence », du moins telle qu'utilisée par la philosophie marxiste orthodoxe, il

36. Ibid., I, p. 149. 
n'y a pas nécessairement de conséquences allant au-delà du truisme P.

Le seul moyen de nier de façon conséquente, et la thèse (i), et le truisme $\mathrm{P}$, serait de nier la possibilité de toute propriété de vérité objective (absolue ou relative) à la connaissance scientifique. Faire ceci tout en continuant à utiliser le terme " connaissance » peut sembler curieux, mais certains philosophes ne se sont pas gênés pour autant. Or, Althusser n'a sûrement pas voulu emprunter cette voie. Néanmoins il y a certainement dans sa pensée des "courants " qui évoquent la position relativiste dans la théorie de la connaissance. On remarque ci-dessous l'utilisation par Althusser du mot « vérité " toujours entre guillemets :

Nous avons en effet montré que la validation d'une proposition scientifique comme connaissance était assurée, dans une pratique scientifique déterminée, par le jeu de formes particulières, qui assurent la présence de la scientificité dans la production de la connaissance, autrement dit par des formes spécifiques qui conferent à une connaissance son caractère de connaissance « vraie ". ${ }^{37}$

. . . la pratique théorique est bien à elle-même son propre critère, contient bien en elle des protocoles définis de validation de la qualité de son produit, c'est-à-dire les critères que la scientificité des produits de la pratique scientifique. Il n'en va pas autrement dans la pratique réelle des sciences : une fois qu'elles sont vraiment constituées et développées, elles n'ont nul besoin de la vérification de pratiques extérieures pour déclarer « vraies » c'està-dire connaissances, les connaissances qu'elles produisent . . . Nous devons en toute rigueur aller plus loin, et refuser d'assimiler plus ou moins indirectement la théorie marxiste de l'histoire au modèle empiriste d'une " hypothèse " aléatoire, dont il faudrait avoir attendu la vérification par la pratique politique de l'histoire, pour pouvoir affirmer la "vérité ". Ce n'est pas la pratique historique ultérieure qui peut donner à la connaissance que Marx a produite, ses titres de connaissance : le critère de la "vérité " des connaissances produites par la pratique théorique de Marx est fourni dans sa pratique théorique elle-même, c'està-dire par la valeur démonstrative, par les titres de scientificité des formes qui ont assuré la production de ces connaissances. ${ }^{38}$

Althusser dit que le critère de scientificité de toute science pleinement constituée et développée, et donc le critère de

37. Ibid., I, p. 82.

38. Ibid., I, p. 71-72. 
" vérité " de ses découvertes, est interne à cette science. L'antirelativiste voudra demander à Althusser si ceci s'applique aussi à des « sciences " telles la théologie traditionnelle, l'astrologie et la phrénologie, qui semblent sûrement capables de se réclamer d'avoir été " constituées et développées " durant plusieurs siècles et de posséder des critères de scientificité internes à elles-mêmes. Comment donc devons-nous décider quelles soi-disant sciences donnent vraiment une connaissance authentique et lesquelles n'en donnent pas $^{39}$ ? Mais ce problème ressemble beaucoup au "problème de la connaissance » des philosophes, problème qu'Althusser rejette :

Nous ne cherchons pas, comme la " théorie de la connaissance » de la philosophie idéologique, à énoncer une garantie de droit (ou de fait), qui nous assure que nous connaissons bien ce que nous connaissons, et que nous pouvons rapporter cet accord à une certaine relation entre le Sujet et l'Objet, la Conscience et le Monde. ${ }^{40}$

Althusser nie-t-il banalement, que ce que nous appelons notre connaissance soit en général garanti, c'est-à-dire infaillible? Sûrement pas, car quelle philosophie traditionnelle n'a pas

39. La suggestion m'a été faite de chercher la réponse althussérienne à cette question dans Lire le Capital, Tome I., p. 66-67, et sur la base de ce qu'y dit Althusser, en tirer l'implication que "l'idéologie est constituée d'un principe de reconnaissance-en-cercle et que la science est constituée d'un développement " ouvert " ». Ceci suppose, toutefois, que toute la pseudo-science est comprise dans ce qu'Althusser appellerait "idéologie ". Certainement, il semble difficile d'inclure l'astrologie et la phrénologie dans ce qu'Althusser dit de l" "idéologie ". Il semble que ce ne soit pas la pseudo-science en général qu'Althusser discute dans ce passage mais plutôt la " théorie de la connaissance " de la philosophie traditionnelle, à laquelle il reproche sévèrement de contenir " la clôture idéologique ", " la clôture préétablie du cercle idéologique ", à cause de sa dépendance des "personnages idéologiques de Sujet et d'Objet " et de "la structure de reconnaissance spéculaire mutuelle, dans le cercle clos de laquelle ils se meuvent ». Il propose de remplacer le « problème de la connaissance » de la philosophie traditionnelle par la " question . . . ouverte, dans son principe même, " du " mode d'appropriation de l'objet réel, spécifique de la connaissance " à travers l'histoire. Cette question " doit être posée . . . en des termes qui forment le concept de la structure de connaissance " pour éviter de "faire de la philosophie cette instance juridique qui, dans les " théories de la connaissance ", légifere pour les sciences au nom d'un droit qu'elle s'arroge elle-même $»$. Ceci semble indiquer que la question historique à savoir, par exemple, comment la physique de la relativité en est arrivée à " approprier l'objet réel ", doit être posée en termes relativistes afin d'empêcher toute remise en question philosophique du statut de la théorie de la relativité en tant que science authentique. De la même façon, l'histoire de l'astrologie et l'histoire de la phrénologie ne devraient-elles pas être examinées en posant la question dans les termes de l'astrologie et de la phrénologie, à savoir comment ces deux " sciences " en sont arrivées à s' " approprier " leurs « objets réels " respectifs, c'est-à-dire l'influence des astres sur nos vies et les corrélats psychologiques de l'ossature crânienne ?

40. Ibid., I, p. 84 . 
reconnu la faillibilité d'au moins la majeure partie de ce que nous appelons notre connaissance ? Nous en sommes donc toujours au problème de déterminer les critères qui nous permettront de répondre à la question de savoir s'il n'y a pas une probabilité quelconque qu'au moins la majeure partie de ce que nous appelons la connaissance soit substantiellement (c'est-à-dire relativement) vraie. Pour un anti-relativiste sérieux qui n'est pas sceptique, il n'y a pas de doute que la réponse à cette question soit affirmative. Mais de son côté, Althusser semble fermement résolu à ne pas poser la question du tout.

Que cet espace du "problème de la connaissance " soit . . . un cercle vicieux . . . toute l'histoire de la " théorie de la connaissance " dans la philosophie occidentale nous le donne à voir, . . . ${ }^{41}$

Mais il n'est pas nécessaire de nier que la théorie de la connaissance soit engagée dans un cercle pour nier que ce cercle soit vicieux. Plutôt, je suggère que c'est précisément la véhémence d'Althusser qui trahit peut-être un trouble de conscience de sa part face au défi posé par le scepticisme/relativisme dans la théorie de la connaissance.

En référence à Descartes, Kant, Husserl et Hegel, Althusser écrit :

Qu'il faille de grands efforts théoriques pour en finir avec toutes les formes de cet empirisme sublimé dans la « théorie de la connaissance " qui domine la philosophie occidentale, pour rompre avec sa problématique du sujet (le cogito) et de l'objet, - et toutes leurs variations - c'est certain. ${ }^{42}$

Ainsi, le problème fondamental dans la " théorie de la connaissance ", selon Althusser, est " sa problématique du sujet et de l'objet ". Qu'entend donc Althusser par la " problématique sujet-objet "? Nous avons déjà vu qu'il suppose que la « catégorie de sujet " signifie la liberté humaine. Est-il admissible de suggérer qu'Althusser s'opposerait à la question, « Comment devons-nous décider quelles prétendues sciences donnent vraiment une authentique connaissance et lesquelles ne le font pas ? ", en disant que la décision ne nous appartient pas puis- 
qu'elle est déjà imposée de façon fatalement inévitable sur notre intellect et donc que nous ne pouvons pas vraiment décider par nous-mêmes de cela ni de quoi que ce soit (même si, sous l'influence de "l'idéologie", nous pouvons penser le contraire)? Cependant, aucun partisan de la science ne peut nier de façon conséquente la possibilité d'éventuellement décider de façon objective (sinon nécessairement infaillible) ce qu'est une science bona fides. Car assurément un tel déni serait incompatible avec une authentique souscription à la thèse de l'objectivité générale de la science. Peut-être Althusser révèle-t-il un malaise envers cette dernière thèse en préconisant une brisure avec la « problématique du sujet et de l'objet $"{ }^{43}$ ? Il se plaint qu'elle :

. . . [escamote], du côté de l'objet la différence de statut entre l'objet de connaissance et l'objet réel, et du côté du sujet la différence de statut entre le Sujet philosophant et le sujet savant, d'une part, entre le sujet savant et le sujet empirique d'autre part. ${ }^{44}$

Je ne saurais comment interpréter la citation suivante autrement que comme l'expression du déterminisme fataliste d'Althusser face à notre "pratique théorique ». Il dit que, selon lui,

La vue [théorique] n'est plus alors le fait d'un sujet individuel, doté d'une faculté du " voir » qu'il exercerait soit dans l'attention, soit dans la distraction; la vue est le fait de ses conditions structurales, . . . ${ }^{45}$

5. Dans cette section finale, je voudrais dévoiler une contradiction, - du genre dont se préoccupent les académiciens, - dans la structure même du système d'Althusser. J'entends par là le genre de " contradiction interne " qui, posée et débattue par les académiciens, a bien servi pendant des années à les sortir du chômage. Je fais allusion à un jugement récemment prononcé par Althusser : "La philosophie n'est pas une science, et elle n'a pas d'objet, au sens où une science a un objet ${ }^{46}$.

Nous avons vu qu'Althusser n'a pas de sympathie pour le concept de vérité relative. Il semble par contre que Lénine, lui,

43. Voir plus loin certaines données indiquant qu'Althusser est de fait capable d'identifier la négation d'un objet à la négation de l'objectivité.

44. Ibid., I, p. 65-66.

45. Ibid., I, p. 25.

46. Althusser, "Lénine devant Hegel " dans Lénine et la pbilosophie, Paris, Maspero, 1972, p. 75 . 
en ait. Mais dans le cas de celui-ci, il n'y a pas de problème, nous dit Althusser, parce que ce n'est pas ce que Lénine voulait vraiment dire.

Lénine annonce dans sa théorie des limites historiques de toute vérité (entendez de toute connaissance scientifique), qu'il pense comme théorie de la distinction de la vérité absolue et de la vérité relative (dans cette théorie sont pensées, sous un seul couple de catégories, à la fois la distinction entre la philosophie et les sciences, et la nécessité d'une théorie de l'histoire des sciences). ${ }^{47}$

Il n'est pas tellement pertinent ici de savoir si Althusser a pu saisir plus correctement que Lénine l'intention de Lénine. Il n'y a pas de substitut à consultation directe et détaillée des textes pour répondre à de telles questions d'interprétations. En ce qui nous concerne ici, il est plus important de prendre note de l'approbation d'Althusser de la thèse, - attribuée à Lénine, voulant qu'il n'y ait pas de vérité philosophique et que la philosophie ne soit donc pas une science.

Je peux proposer qu'Althusser, en niant que la philosophie ait un objet, entend par là que la philosophie n'est pas objective : c'est-à-dire qu'il n'y a aucune réalité dont la philosophie puisse traiter (même " indirectement ») et dont, par la caractérisation correcte, la philosophie puisse espérer se constituer en science objective. En d'autres mots, Althusser est un " non-objectiviste » en philosophie. Ses raisons pour le non-objectivisme quant à la philosophie sont les mêmes que celles invoquées par les " nonobjectivistes » en éthique.

Toute philosophie exprime une position de classe, une " prise de parti » dans le grand débat qui domine toute l'histoire de la philosophie, le débat entre l'idéalisme et le matérialisme. ${ }^{48}$

De fait, cette thèse revient à affirmer que, pour l'essentiel, la philosophie n'a pas vraiment d'bistoire. Qu'est-ce qu'une histoire qui n'est que la répétition du heurt de deux tendances fondamentales ? Les formes et les arguments du combat peuvent varier, mais si toute l'histoire de la philosophie n'est que l'histoire de ces formes, il suffit de les réduire aux tendances immuables qu'elles représentent pour que la transformation de ces formes devienne une sorte de jeu pour rien. ${ }^{49}$

47. Lénine et la philosophie, p. 37.

48. "Lénine devant Hegel ", Op. cit., p. 75.

49. Lénine et la philosophie, p. 42-43. 
Lénine [préconise] . . . une thèse matérialiste qui est conjointement une thèse d'existence (matérielle) et d'objectivité (scientifique). ${ }^{50}$

Lénine . . déclare qu'on ne peut pas démontrer les principes derniers du matérialisme, pas plus qu'on ne peut démontrer (ni réfuter . . . ) les principes de l'idéalisme. On ne peut les démontrer car ils ne peuvent être l'objet d'une connaissance, entendons d'une connaissance comparable à celle de la science qui démontre les propriétés de ses objets.

La philosophie n'a donc pas d'objet. Mais, tout se tient. S'il ne se passe rien dans la philosophie, c'est justement parce qu'elle n'a pas d'objet. S'il se passe en effet quelque chose dans les sciences, c'est qu'elles ont un objet, dont elles peuvent approfondir la connaissance, ce qui leur donne une bistoire. ${ }^{51}$

Jusqu'ici Althusser ressemble tout à fait à un positiviste logique. Il attribue à Lénine toutes les positions citées plus haut et compare défavorablement Engels à ce dernier pour avoir pensé que la philosophie a un objet ${ }^{52}$ et n'avoir pas reconnu la nature de la philosophie en tant que lutte politique ${ }^{53}$.

Ce qui se joue dans la philosophie, au travers des catégories dernières, qui commandent tous les systèmes philosophiques, c'est donc le sens de cette hiérarchie, le sens de cette mise d'une catégorie en position de domination, c'est dans la philosophie quelque chose qui fait irrésistiblement penser à une prise de pouvoir ou à une mise au pouvoir. Philosophiquement, nous devons dire : une mise au pouvoir est sans objet. Une mise au pouvoir, est-ce encore une catégorie purement théorique? Une prise de pouvoir (ou mise au pouvoir) est politique, elle n'a pas d'objet, elle a un enjeu, justement le pouvoir, et un objectif : les effets du pouvoir. ${ }^{54}$

... toute philosophie consiste dans le tracé d'une ligne de démarcation majeure par quoi elle repousse les notions idéologiques des philosophies qui représentent la tendance opposée à la sienne ; l'enjeu de ce tracé, donc de la pratique philosophique, est la pratique scientifique, la scientificité. ${ }^{55}$

... la philosophie serait la politique continuée d'une certaine manière, dans un certain domaine, à propos d'une certaine réa-

50. "Lénine devant Hegel ", Op. cit., p. 84.

51. Lénine et la philosophie, p. 35.

52. Ibid., p. 45-48.

53. Ibid., p. 54.

54. Ibid., p. 46.

55. Ibid., p. 50. 
lité. La philosophie représenterait la politique dans le domaine de la théorie, pour être plus précis : auprès des sciences, - et vice-versa, la philosophie représenterait la scientificité dans la politique, auprès des classes engagées dans la lutte des classes. ${ }^{56}$

Le moment est venu de mettre à jour la contradiction du genre académique dans le système d'Althusser. (On qualifie la démonstration d'une telle contradiction au sein d'un système de pensée comme simple « exercice académique » parce qu'une telle démonstration ne peut prétendre avoir une importance significative que dans la mesure où le système est candidat viable à être accepté comme vrai.) La philosophie matérialiste, dit Althusser, est pro-scientifique, mais est en soi non-scientifique, c'est-àdire non-objective (c'est justement ce que la philosophie positiviste logique dit d'elle-même, bien sûr, lorsqu'elle est attentive). Les raisons pour lesquelles elle n'est pas objective, selon lui, sont a) qu'elle est impossible à prouver et b) qu'elle est partisane. Une telle philosophie partisane, même politiquement partisane vis-à-vis la lutte de classe prolétarienne, exprime donc sûrement une volonté. Ainsi, selon Althusser, elle ne peut pas être autrement qu'idéologique et tous ceux qui l'approuvent tombent alors dans cette mesure sous l'influence de l'idéologie. Par contre, il s'ensuit que puisqu'il est dit philosophie proscientifique, dont anti-idéologique, le matérialisme althussérien conséquent, ne peut pas manquer de prôner son propre rejet.

Peut-on deviner quelle serait la réponse d'Althusser ? Son objection à cet argument serait peut-être qu'il escamote

la différence de statut entre le Sujet philosophant et le sujet savant, d'une part, et entre le sujet savant et le sujet empirique d'autre part.

Quelle différence?

Department of Philosophy

University of Toronto

56. Ibid., p. 54 . 